Berthoz, S., Lalanne, C., Crane L. \& Hill E.L. (2013). Investigating emotional impairments in adults with autism spectrum disorders and the broader autism phenotype. Psychiatry Research, 208 (3), 257-264. (Elsevier)

DOI: 10.1016/j.psychres.2013.05.014

\title{
Investigating emotional impairments in adults with autism spectrum disorders and the broader autism phenotype
}

Sylvie Berthoz $\mathrm{PhD}$ 1,2,3

Christophe Lalanne $\mathrm{PhD}^{1,3,4}$

Laura Crane $\mathrm{PhD}^{5,6}$

Elisabeth L Hill $\mathrm{PhD}^{*} 5$

${ }^{1}$ Institut National de la Santé et de la Recherche Médicale (INSERM U669), Paris, France

${ }^{2}$ Institut Mutualiste Montsouris (IMM), Paris, France

${ }^{3}$ Paris-Sud and Paris-Descartes Universities, Paris, France

${ }^{4}$ AP-HP, Department of Clinical Research, Saint-Louis Hospital, Paris, France

${ }^{5}$ Goldsmiths, University of London, UK

${ }^{6}$ London South Bank University, UK

*Address correspondence to: Dr Elisabeth L. Hill, Department of Psychology, Goldsmiths, University of London, London, SE14 6NW, UK; e.hill@gold.ac.uk

\begin{abstract}
There is an increasing interest in the socio-affective atypicalities observed in adults with autism spectrum disorder (ASD). The aim of this study was to further explore emotional responsiveness in adults with ASD using well-validated self-reports of alexithymia and extend these with consideration of anhedonia, and to determine whether these features are part of a broader autism phenotype. Thirty-eight adults with ASD, 87 parents of ASD individuals and 47 typical controls completed the Autism Spectrum Quotient, the 20-item Toronto Alexithymia Scale and the Bermond-Vorst Alexithymia Questionnaire, as well as the Chapman Physical and Social Anhedonia Scales. The ASD group differed from controls and parents on most measures, with the exception of physical and social anhedonia, relative to parents. Parents differed from controls on social anhedonia, and a higher proportion of parents were classed as alexithymic, relative to controls. Cluster analysis revealed that some parents share more similarities with ASD participants than with controls. The results suggest that socio-affective impairments are characteristic of ASD, and feature as part of the broader autism phenotype.
\end{abstract}

Keywords: alexithymia, social anhedonia, physical anhedonia, anxiety, autism spectrum disorder, depression, psychometric 


\section{INTRODUCTION}

Between the triad of symptoms characteristic of autism spectrum disorders (ASD), socio-affective impairments are the only deficits specific to ASD, and are thus likely 'to be the most informative with respect to modelling the pathophysiology of the disorder' (see Schultz, 2005, p. 125). Psychometric studies have highlighted that those with ASD not only have difficulties in interpersonal skills such as few friendships, little emotional connectedness, empathy and social enjoyment (e.g., Baron-Cohen et al., 2001, Baron-Cohen and Wheelwright, 2003, 2004; Rogers et al., 2007; Chevallier et al., 2012a; Calder et al., 2012), but also have difficulties in processing their own emotions including poor emotional introspection, clarity, expression and regulation (e.g., Hill et al., 2004; Berthoz and Hill, 2005; Konstantareas and Stewart, 2006; Szatmari et al., 2008; Petrides et al., 2011; Rieffe et al., 2011; Samson et al., 2012). Hedonic abnormalities from sensitive arousal have also been noted (e.g., Baranek et al., 2006; Baron-Cohen et al., 2009).

There is increasing interest in determining whether these emotional and hedonic features should be considered core factors underlying the social and communication deficits of ASD with findings from quantitative, behavioural studies being supplemented in recent years by findings of neuroimaging research. This can be exemplified with alexithymia, as there is now a converging line of evidence that it may account to a great extent for the behavioural and cerebral responses to social and emotional stimuli in those with ASD. For instance, Kätsyri et al. (2008) assessed the processing of emotional facial expressions (EFEs) in adults with Asperger syndrome and healthy controls by varying the perceptual quality of EFEs. Compared to controls, the group with Asperger syndrome had higher alexithymia scores and was less accurate in labelling strongly degraded EFEs. However, this latter group effect was no longer significant when alexithymia scores were entered as a covariate. More recently, Bird et al. (2011) investigated the potential impact of alexithymia on joint attention deficits in ASD adults. Whereas the ASD pattern of attention allocation was not related to the severity of their autistic symptoms (ADOS scores), alexithymia scores were found to be negatively correlated with the number of fixations to the eye region (relative to the mouth region) (see also Lombardo et al., 2007). At the cerebral level, this issue is well illustrated in a study which investigated the neural correlates of empathy for pain in adults with ASD and healthy controls: Bird et al. (2010) not only showed that, in both groups, the higher the level of alexithymia the lower the empathy-related activity in the insula when the partner received pain, but also that there were no group differences in brain activity when alexithymia scores were entered as a covariate (see also Silani et al. 2008). Thus, extending our knowledge of socio-affective abnormalities such as alexithymia, as well as anhedonia (i.e., the inability to experience pleasure) in ASD could not only explain previously inconsistent results in our understanding of ASD (Bird et al., 2011; see also Bernhardt and Singer 2012), but also help to tailor therapeutic interventions for this group.

Moreover, in ASD as for other psychiatric disorders that are phenotypically complex and highly heritable, there is a growing consensus that research into the aetiology of the condition could benefit from the investigation of heritable markers (or endophenotypes, see Gottesman and Gould, 2003), that is traits that are also present in unaffected biological relatives of ASD but exhibited to a lesser degree (Adolphs et al., 2008; Geschwind, 2009; Sucksmith et al., 2011). In this domain of research, the so-called Broader Autism Phenotype (BAP), mild social and communication impairments have been suggested to be likely 'the most familial of autism-related traits' (Gerdts and Bernier, 2011, p.14). Milder phenotypes in socio-affective and personality traits have been reported in biological relatives of individuals with ASD, with consistent results from self-reports, clinical interviews and cognitive evaluations (see Gerdts and Bernier, 2011, for a recent review). Yet, to our knowledge, only one study has examined whether alexithymia is part of the traits that tend to aggregate in ASD relatives (Szatmari et al., 2008), and no study has explored whether this is also the case for anhedonia. 
The aim of this study was to further explore traits related to emotional responsiveness (alexithymia and anhedonia) in adults with ASD relative to a group of typical adults and to determine whether they constitute key components of the broader autism phenotype by exploring these traits in first-degree relatives of individuals with ASD. In this way the study will both develop, and extend, our understanding of socio-affective impairments in ASD.

\section{METHODS}

\subsection{Participants}

A total of 172 people participated in the study: 38 high-functioning adults with ASD (mean age $=$ 35.5 years, $\mathrm{SD}=13.3 ; 63 \%$ male), 87 parents of individuals with ASD (mean age $=43.6$ years, $\mathrm{SD}$ $=7.3 ; 28 \%$ male) and 47 typical adult controls (mean age $=33.7$ years, $\mathrm{SD}=11.7 ; 62 \%$ male). The adults with ASD had all received a formal diagnosis (by expert clinicians) of either Asperger syndrome $(n=32)$ or autism $(n=6)$ according to DSM-IV-TR criteria (American Psychiatric Association, 2000). In line with a range of studies with adults (e.g., Crane et al., 2012; Heaton et al., 2012), Autism Spectrum Quotient scores (AQ; Baron-Cohen et al., 2001) were used as a further support of their clinical diagnoses and provide a measure of autistic traits for the three groups (NB. The AQ is not a diagnostic tool, see below, and there are ambiguities in its psychometric properties. However it is used here to allow comparison of autistic traits with variables of interest, and has been shown to be associated with, and a predictor of, various characteristics in the typical population, see for example, Freeth, et al., 2012; Poljac et al., 2012). 13\% of the ASD sample reported an additional diagnosis, specifically: one participant reported an additional diagnosis of $\mathrm{ADHD}$, one of dyslexia, two of developmental coordination disorder (DCD, sometimes referred to as 'dyspraxia') and one of ADD and DCD. Nineteen (50\%) of the adults with ASD had participated in a previous study investigating cognitive processing of emotions in people with ASD (Hill et al., 2004). The other 19 completed questionnaires were received from participants who had been recruited via various specialized support groups and community centres in the UK. Parents of people with ASD were recruited either via those included in the adult ASD group or were the parents of children/adults involved in other projects that were running in our laboratory. Of these, 18 (20.68\%) had participated in the previous study on this topic (Hill et al., 2004). The typical adult control group was recruited predominantly from the subject pool at the Institute of Cognitive Neuroscience (UK) and from local community centers. No participant in either the parent or control groups reported having received a diagnosis of ASD.

\subsection{General procedure}

Questionnaires were given or sent out to participants with full instructions and stamped addressed envelopes for their return (including information relating to informed consent). Participants were encouraged to contact one of the authors $(\mathrm{EH})$ if they had questions concerning completion of the questionnaires, which none of them did.

\subsection{Data analyses}

Statistical analyses were performed using R 2.11.1. We considered a Type I error rate of 5\% and provided $95 \%$ confidence intervals (relying on asymptotic distribution or bootstrap with adjusted percentiles). Comparisons of means were achieved by way of ANOVA, followed by post-hoc pairwise comparisons. Two-way contingency tables were analysed with chi-square tests. Latent profile analysis was carried out using parametric mixture modelling, as implemented in the R MCLUST package (Fraley and Raftery, 2002, 2006).

\subsection{Self-report Measures}


- The Autism Spectrum Quotient (AQ) (Baron-Cohen et al., 2001) is a 50-item questionnaire measuring traits associated with the autism spectrum. Participants rate their own behaviour in five subscales: social skill, attention switching, attention to detail, communication and imagination on a 4-point scale. AQ total and subscale scores can be calculated. Total scores can be classified into one of four categories: Typical (<23), Broader Autism Phenotype (BAP; 23-28), Medium Autism Phenotype (MAP; 29-34) or Narrow Autism Phenotype (NAP; > 35) (Wheelwright et al., 2010). People with the NAP have a large number of traits associated with the autism spectrum and most, but not all, will have a diagnosis of ASD. Individuals with the MAP or BAP are unlikely to require clinical intervention, but these phenotypes are commonly observed in parents of individuals with ASD (Wheelwright et al., 2010).

- Levels of depressed mood were assessed using the 21-item Beck Depression Inventory (BDI) (Beck et al., 1961) in which individuals respond to statements on the basis of how they have felt over the past week. The BDI provides an indication of the presence of a depressive episode at the time of the study. Scores were totalled (range $=0-63$ ) and considered as a continuous variable, as well as being categorised using established cut-off scores to assign participants to a group of those considered to exhibit non-depressed functioning (0-10), dysphoria (11-14), dysphoria/depression (15-19) or clinical depression (20-63) (Beck et al., 1988). Missing values ( $n=14$, with the number of missing responses less than $25 \%$ of total number of items on the BDI) were imputed using individual mean scores. The internal consistency of the full scale was satisfactory (Cronbach's $\alpha=0.878$, with $95 \%$ bootstraped CIs $[0.841 ; 0.906]$ ).

- Levels of anxiety were assessed using the State-Trait Anxiety Inventory Form Y (STAI-Y) (Spielberger, 1983). The state portion of the scale (STAI-S; 20 items) asks respondents to report the extent of their anxiety at present, while the trait scale (STAI-T; 20 items) asks respondents to indicate the intensity of their anxiety in general. The items are rated by the respondent on a fourpoint Likert scale. Responses were totalled (range $=20-80$ ) for each of the two parts of the scale. The scores for the state and trait anxiety scales were considered as two separate continuous variables. Internal consistency was good for each subscale (STAI-S, $\alpha=0.946$, [0.933;0.956]; STAI$\mathrm{T}, \alpha=0.947,[0.932 ; 0.957])$.

- The 20-item Toronto Alexithymia Scale (TAS-20) (Bagby et al., 1994a,b) investigates three dimensions: difficulty identifying feelings (DIF), difficulty describing feelings (DDF) and externally oriented thinking (EOT). The items are rated by the respondent on a five-point Likert scale. Responses were totalled (range $=20-100$ ) and considered as a continuous variable, as well as being categorised using previously established cut-off scores (Bagby et al., 1994a) to assign participants to one of three groups: not alexithymic (20-51), intermediates (52-60) or alexithymic (61-100). The internal consistency of the full scale was satisfactory (TAS-20 total $\alpha=0.889$, [0.862;0.910]); DIF $\alpha=0.883$, average inter-items correlation $r=0.519$; DDF $\alpha=0.842, r=0.515$; and EOT $\alpha=0.660, r=0.196$ ).

- The Bermond Vorst Alexithymia Questionnaire-B (BVAQ-B) (Vorst and Bermond, 2001) includes 20 item investigating five dimensions of alexithymia: verbalising emotional experiences (B1, equivalent to DDF), fantasising (i.e., daydreaming and fantasies, B2), identifying emotions (B3, equivalent to DIF), emotionalising (i.e., the proneness to be aroused by emotion inducing events, B4) and analysing one's own emotional states and reactions (B5, equivalent to EOT). The items are rated on a five-point Likert scale. Responses were totalled (range $=20-100)$ and considered as a continuous variable. Missing values $(n=2)$ were imputed using the BVAQ-B neutral response category (i.e., I neither agree, nor disagree). The internal consistency of the full scale was in the acceptable range $(\alpha=0.755,[0.703 ; 0.806])$, and varied between $0.527(\mathrm{~B} 5, r=0.219)$ and 0.688 (B1, $r=0.355$ ) when considering individual subscales. We followed the methodology reported by Vorst and Bermond (2001) and computed three scores based on the BVAQ-B subscales. The BVAQ-B standard total score equals the sum of all five subscales. The BVAQ-B Cognitive score corresponds to the sum of the scores on the Identifying, Verbalising and Analysing subscales (B3, 
B1, B5, respectively; equivalent to TAS-20 total). It showed acceptable internal consistency $(\alpha=0.766,[0.712 ; 0.818]), r=0.213)$. Its concurrent validity with the TAS-20 total score was acceptable $(r=0.826, P<0.001,95 \%$ CI $[0.772 ; 0.868])$. The BVAQ-B Affective score corresponds to the sum of the scores on the Fantasising and Emotionalising subscales (B2 and B4, respectively). It had lower internal consistency ( $\alpha=0.608,[0.504 ; 0.692], r=0.162)$.

- The Physical Anhedonia Scale (PAS) (Chapman et al., 1976) is a 61 item (true or false) questionnaire assessing the degree to which individuals are rewarded by physical sensations such as touch, feeling, movement, eating, smell and sound. It measures a lifelong, rather than a transient, difficulty in the ability to experience pleasure. Scores were totaled (range $=0-61$ ) and considered as a continuous variable as well as being categorised using established cut-off scores to assign participants to a group of those considered to exhibit physical anhedonia $(\geq 18)$ or not $(\leq 17)$ (Assouly-Besse et al., 1995). The internal consistency of the PAS was satisfactory $(\alpha=0.937$, $[0.923 ; 0.950])$.

- The Revised Social Anhedonia Scale (SAS) (Eckblad et al., 1982) is a 40 item questionnaire measuring social withdrawal, a lack of interest in social relationships and/or lack of pleasure derived from interpersonal relationships. It was designed to tap a personality trait. The test is set out in the same way as the PAS, as is the scoring. Scores were totaled (range $=0-40$ ) and considered as a continuous variable as well as being categorised using established cut-off scores to assign participants to a group of those considered to exhibit social anhedonia $(\geq 12)$ or not $(\leq 11)$ (Assouly-Besse et al., 1995). The internal consistency of the SAS was satisfactory $(\alpha=0.935$, $[0.920 ; 0.947])$.

\section{RESULTS}

The gender distribution differed significantly between the three groups $[\chi 2(2)=21.04, P<0.001]$, with a larger proportion of males in the ASD group and a larger proportion of females in the Parent group. This difference is unsurprising (e.g., Baird et al., 2006). There was also a significant difference in the age of the groups $\left[F(2,169)=17.65, P<0.001, \eta_{\mathrm{p}}{ }^{2}=.17\right]$. Pairwise t-tests (with Holm's adjustment for multiple comparisons) confirmed that this was due to the Parent group being significantly older than both the ASD and Control groups, but these latter groups did not differ significantly from each other $[P=0.431]$.

Table 1 displays the mean scores for each group on the questionnaires. In each case, we tested between-group differences using an $F$-test (with 2 and 167 degrees of freedom) comparing the extra-variance accounted for by questionnaire scores with a base model including age and gender only. When the overall statistic proved to be significant, we further tested whether there were significant differences between the Parent and ASD groups vs. Controls (post-hoc Tukey's tests, adjusted on the aforementioned covariates). All but one (BVAQ-B Affective) questionnaire yielded significant between-group differences, these differences being somewhat more marked between the ASD and Control groups, than between the ASD and Parent groups. It is worth noting that Controls and Parents did show significant differences on the PAS scale (with higher scores among the Parents), whereas this was not the case between the ASD and Parent groups. When controlling for current dysphoric affect scores (BDI and STAI-S), differences between the ASD and Control groups remained significant at the 5\% level for all trait measures, and the difference between the Control and Parent groups for PAS scores also remained (Supplementary Table 1). However, this analysis changed two between-group comparisons. First, whereas the ASD vs. Control group comparison did show significantly different SAS scores $[P=0.004]$, this was no longer the case between the ASD and Parent groups $[P=0.134]$. Second, we identified a significant between-group effect for the BVAQ-B Affective scores $\left[P=0.029 ; \eta_{\mathrm{p}}{ }^{2}=0.04\right]$, which was explained by a significant difference between the ASD and Control groups $[P=0.023]$, but no difference between the ASD and Parent groups or between the Control and Parent groups. 
[insert Table 1 and Figure 1 about here]

For those test measures where cut-off scores were used, the percentage of each group falling into each category is shown in Table 2. Interestingly, $21.8 \%$ of Parents $(n=19)$ were categorised as 'atypical' according to AQ categories, whereas only $8.5 \%$ of Controls $(n=4)$ were categorised as such [two-sample test for equality of proportions, $\chi 2(1)=5.57, P=0.018]$.

[insert Table 2 about here]

A Principal Components Analysis was used to summarise the structure of the correlation matrix of all trait scores (AQ, TAS-20, BVAQ-B Cognitive and Affective subscales, SAS, PAS, STAI-T), considering gender, clinical group, BDI, STAI-S and BVAQ-B total scores as supplementary or illustrative variables. It highlighted two main components (71.9\% of the total variance, see Supplementary Figure), with a large contribution of AQ, BVAQ-B Cognitive, TAS-20 and PAS and SAS scores (correlations ranging from 0.38 to 0.83 , see Table 3 ) on the first dimension.

As illustrated in Figure 1, the Parents were found to lie in between Controls and participants with ASD. The preceding results suggest that some Parents are more likely to be considered at risk of meeting cut-offs/criteria for a variety of socio-affective traits than Controls and that the distinction between those with ASD and the Parents may not be as clear as the distinction between those with ASD and the Control group.

To assess the extent to which those with ASD and the Parents may overlap on the instruments used throughout this study, we performed two iterations of a Latent Profile Analysis (LPA) with the aim of defining a mixture of class-specific densities. LPA (or 'model-based clustering') assumes that the population is composed of different unobserved groups, or latent classes, and that the joint density of all manifest variables is a mixture of this class-specific density (Lazarfield and Henry, 1968). We used MCLUST to find the optimal model as defined by minimal BIC criterion when spanning the models' space ( 1 to 9 components or clusters, 10 kinds of constrained variance-covariance matrix). The following ten trait scores were considered: the five AQ subscores, STAI-T, TAS-20, BVAQ-B (BVAQ-B Total score in LPA version 1; BVAQ-B Cognitive score in LPA version 2), PAS and SAS. These trait scores were first residualised with respect to age, gender, BDI and STAI-S to remove conditional associations. For both versions of the LPA, the best fitting model included four clusters with diagonal but equally shaped variancecovariance matrices for the 10 trait scores. The cross-classification of cluster membership with group (Table 4, section I) indicated that participants with ASD were distributed across two clusters, with one cluster being composed of $68 \%$ of them (together with $28 \%$ of Parents and $4 \%$ of Controls, cluster D), while the other cluster included $37 \%$ of participants with ASD, $44 \%$ of Parents and $19 \%$ of Controls (cluster C). Of note, using the BVAQ-B Cognitive subscale in place of BVAQ-B total score did not alter these results (Table 4, section II); the same clusters were observed with the ASD participants (C and D), and only three participants were found to differ with respect to their cluster C or D membership, leaving only ASD and Parent participants in cluster D.

\section{[insert Table 3 about here]}

As evidenced in Figure 2 (top), which shows the distribution of AQ scores according to clinical and cluster membership, individuals from cluster $C$ (which mixes up all three groups) were more likely to span the entire range of the scale, whereas individuals from cluster $\mathrm{D}$ (which was more geared towards a majority of ASD participants along with Parents) clearly overlapped with 
the observed AQ total scores distribution for ASD participants. This suggests that some parents share more similarities with ASD participants in this particular cluster.

As supported by the distribution of standardized scale scores in each cluster (Figure 2, Bottom), a clear opposition appears to exist between cluster A (all mean scores below the overall mean, by about $0.5 \mathrm{SD}$ ) and cluster D (all mean scores above the overall mean, by about $1 \mathrm{SD}$ ), while standardized scores observed in cluster $\mathrm{C}$ on the alexithymia and anhedonia scales are largely below those observed in cluster $\mathrm{D}$. This shows that the segregation between clusters $\mathrm{C}$ and $\mathrm{D}$ cannot be explained by differences in AQ scale scores only. However, in cluster B, only PAS and SAS scores are above the mean, suggesting that participants in this cluster have a stronger tendency to experience anhedonia than others.

[insert Table 4 and Figure 2 about here]

\section{DISCUSSION:}

The primary purpose of the current study was to use self-report questionnaires to explore a range of traits related to emotional responsiveness in adults with ASD, relative to a group of typical adults. Our secondary aim was to determine whether some of these traits might constitute endophenotypic markers of the condition (i.e., part of a broader autism phenotype). The total sample recruited was substantial and the ASD group was representative of the larger ASD population in terms of both sex ratio and AQ scores (e.g., Baron-Cohen et al., 2001; Baird, et al., 2006; Wheelwright et al., 2010).

When comparing adults with and without ASD, clear group differences were found across the range of socio-affective impairments measured, with the ASD group endorsing increased numbers of each characteristic relative to their peers. In terms of depressive symptoms, around $63 \%$ of the current ASD sample endorsed enough symptoms to be considered dysphoric or to have clinically significant levels of depression, which is similar to previous reports in ASD adults (e.g., Hill et al., 2004). This proportion is higher than recent findings from younger ASD participants (e.g., Cederlund et al., 2010), although the latter study applied different cut-offs to those typically used for the BDI and these required greater numbers of BDI items to be endorsed in order for the 'dysphoric' label to be applied. In our study, the key objective was not to study the comorbidity between ASD and clinical depression, but to estimate current dysphoric affects (as measured by the BDI and STAI-S), as these can bias responses on self-report measures of alexithymia and anhedonia. In the present study, accounting for current levels of dysphoric affects provided additional evidence of a combination of socio-affective trait atypicalities in ASD.

With respect to alexithymia, more than half of the ASD sample was categorized as alexithymic (i.e., TAS-20 scores). The type of alexithymia is of interest since different subtypes of alexithymia have been proposed (Bermond, 1997; Bermond et al., 2007) depending on the balance between the five core alexithymic dimensions that are modelled in the BVAQ. Initially, two subtypes were distinguished: 'alexithymia type I', characterised by severe reductions in both emotionalising and emotion-accompanying cognitions, and 'alexithymia type II', characterised by the unreduced (full-blown) presence of the emotional feeling (i.e., low BVAQ Affective scores) in combination with severe reductions in the cognitions normally accompanying the emotional feeling (i.e., high BVAQ Cognitive scores). Two additional subtypes were subsequently introduced (Bermond, et al., 2007): 'alexithymia type III', characterised by low affective and high cognitive capacities, and 'lexithymic type' (i.e., the antonym of alexithymia), characterised by high affective and high cognitive capacities. The finding of low BVAQ-B Cognitive scores in the current research confirms the presence of Alexithymia Type II in ASD (Hill et al., 2004), but taking current affectivity into account also highlighted a group effect for the BVAQ-B Affective scores, suggesting that ASD might rather be characterized by Alexithymia Type I. To further explore this issue, and to use Bermond and colleagues' (2007) scoring procedure to determine alexithymia type, 
future studies should use the long (40 item) version of the BVAQ (see Berthoz et al., 2011, for a detailed description). This is of critical importance, given that alexithymia has been found to underlie several social and emotional characteristics of ASD such as reduced eye fixation (Bird et al., 2011) and impaired recognition of facial emotions (Kätsyri et al., 2008), rather than these being characteristics of ASD per se.

With respect to anhedonia, Rawlings and Locarnini (2008) reported a significant, positive correlation between total AQ score and the Introvertive Anhedonia (self-report) scale of the O-LIFE (Mason and Claridge, 2006), suggesting an association between autistic traits and anhedonia in the general population. Of interest too, cerebral responses to sensory stimulation have been linked with social and communication symptoms of ASD (Cascio et al., 2012) or with the level of autistic traits (AQ scores) in neurotypical individuals (Voos et al., in press). In the present study, anhedonia and autistic-traits scores were positively associated (more strongly with social anhedonia). Moreover, our findings provide the first exploration of hedonism using self-reports in adults with ASD, extending recent similar findings reported in adolescents with ASD (Chevallier et al., 2012a), and confirming early clinical descriptions of a lack of hedonism in ASD (e.g., Kanner, 1943). While Chevallier and colleagues (2012a) only identified a group difference with regards to social anhedonia in their adolescent sample, our adult sample with ASD reported significantly higher levels of both social and physical anhedonia in comparison to their peers. This could to be due to differences in the instruments used to measure anhedonia. However, the finding that both adolescents and adults with ASD were socially anhedonic is consistent with the social motivation hypothesis of this disorder, which suggests that individuals with ASD do not experience social stimuli as rewarding (e.g., Chevallier et al., 2012b; Khols et al., 2012). With respect to the other measure of anhedonia, the high incidence (81.6\%) of physical anhedonia in the ASD group appears to contradict reports of sensory hypersensitivity in ASD (e.g, Rogers \& Ozonoff, 2005; Minshew \& Hobson, 2008; Baron-Cohen et al., 2009). Yet, these findings could be reconciled considering that hypersensitivity in ASD often evokes particularly strong positive or negative feelings (e.g., Robledo et al., 2012; Cascio et al., 2012) whereas in the present study anhedonia was measured using only yes/no responses (as is standard in the PAS). Further studies using response modalities that vary from 'very unpleasant', through 'neutral' to 'very pleasant' could provide further information in this regard. In addition, there is an increasing number of studies in clinical conditions with marked anhedonia (depressive disorders and schizophrenia) which reported an association between the level of physical anhedonia and abnormal response of brain regions that have been critically involved in emotional and social stimuli processing (e.g., amygdala, OFC, ACC, ventromedial PFC) (e.g., Harvey et al., 2010; Dowd and Barch, 2012; Stuhrmann et al., 2012). As recently discussed for alexithymia (Bird et al., 2011; see also Bernhardt and Singer 2012), the question as to whether anhedonia is a core feature that could explain previous neuroimaging findings in ASD should be addressed in future studies. Moreover, hedonic experience and motivation are dissociable constructs subserved by different neurobiological systems (Berridge and Robinson, 2003). As the Chapman anhedonia scales tend to contain items relevant to both constructs (their elaboration predate the determination of the neural circuitry of reward responsiveness), our study does not inform the question of whether those with ASD have only poor hedonic experience, or motivation, or both. This is a topic that has recently received increased attention in ASD (e.g., see Kohls et al., 2012), and future studies using instruments that carefully distinguish between these deficits (such as the Clinical Assessment Interview for Negative Symptoms scale; Horan et al., 2011) are needed.

The secondary objective of this research was to explore whether alexithymia and anhedonia, and not only autism-related traits, as measured by the AQ, could be considered as endophenotypic markers of a broader autism phenotype. Regarding the traits assessed within this research, the boundaries between the ASD and Parent groups were less clear than those between the ASD and Control groups. Of the Parent group, $21.8 \%$ did not fall in the Typical AQ category whereas this was the case for $8.5 \%$ of the Control participants, none of whom belonged to the MAP or NAP 
categories. With respect to alexithymia, despite no group difference overall, a significantly greater percentage of Parents were classed as alexithymic (TAS-20 criteria) than Controls; a finding consistent with previous results confirming alexithymia as a feature of the broader autism phenotype (Szatmari et al., 2008). Regarding the measures of both physical and social anhedonia, the Parents scored mid-way between the ASD and the Control groups, with no significant difference between the ASD and Parent groups. Further, using Latent Profile Analysis accounting for a wide range of socio-affective traits (and not just AQ scores), we were able to demonstrate that out of the four observed clusters, the two that were composed of ASD participants also encompassed a greater proportion of Parents than of Controls. Of interest, we observed a transition from cluster A or B (which were composed of Parents and Controls only) to cluster $\mathrm{C}$ (which mixed up the three groups) that somewhat matches the Typical AQ cut-off score, whereas the distinction between clusters $\mathrm{C}$ and $\mathrm{D}$ seems to be at the boundary between BAP/MAP and MAP/NAP.

Regarding the limitations of the present research, we did not confirm participants' diagnoses of ASD directly during the current study. This is a key difficulty for research into ASD across the lifespan given the paucity of adult appropriate diagnostic tools, practical issues surrounding complete paperwork or lack of a parent to confirm childhood diagnosis or symptomatology, as well as ethical issues of rediagnosing individuals. However, all participants had previously received a formal diagnosis from a clinical professional that was further supported on the basis of scores on the AQ in all but one case (this is higher consistency in terms of high AQ scores than reported in Baron-Cohen et al., 2001). This approach is used widely in studies of adults with ASD since although other quantitative estimations of ASD diagnoses are available (Lord et al., 1999), these become less specific when used with adults (relative to children), as well as with individuals at the higher functioning end of the autism spectrum (Lord and Risi, 1998). Future prospective or longitudinal studies may overcome this.

A further concern may be the lack of an IQ evaluation of participants. This was not possible in the context of the current study, however given that the sample included participants whose IQ performance matched those of a proportion of the control group who had participated in cognitivebehavioural studies for our research group in the past (e.g., Hill and Bird, 2006; Heaton et al., 2012), that other participants were recruited from similar sources to that portion of the sample and that our findings are consistent with published studies that have reported IQ and TAS-20 scores (e.g., Bird et al., 2011), we are confident that the ability levels of the participant groups were at least appropriate for the demands of the study. Moreover, our study provides a proof of concept for further more detailed investigations.

As with all questionnaire studies, issues relating to self-reports and response biases apply, which could have affected the observed pattern of results. Additional concerns over the accuracy of self-report in individuals with ASD should also be considered, as exemplified by previous (conflicting) reports of disagreement between the number of symptoms of anxiety and mood disorders endorsed by parents, teachers and those with ASD (e.g., Hurtig et al., 2009; Lopata et al., 2010; Mazefsky et al., 2010). To this end, it is particularly reassuring that self-report measures of the traits that were assessed in the present study are associated with performance and neuroimaging measures in ASD samples. For example, Kätsyri and colleagues (2008) found that the level of selfreported alexithymia correlated with emotional facial expression decoding performance, whilst Silani and colleagues (2008) reported an association between the level of self-reported alexithymia and empathy - which were related to brain activity in the anterior insula - and Bird et al. (2011) observed that greater self-reported alexithymia was associated with reduced attentional resources allocation to relevant facial areas. These studies suggest that alexithymia, as measured by selfreports, could be a promising emotional endophenotypic marker in ASD. To build on these, studies utilizing observer-rated evaluations (e.g., Berthoz et al., 2007) should be conducted to investigate this further, as well as behavioural and neuroimaging studies using a composite battery (including measures of emotional intelligence and emotion regulation). 
Table 1. Mean scores (SD, range) on each test measure separately. The last three columns correspond to post-hoc multiple comparisons (Tukey's method, adjusted for age and gender), with test statistic and $P$-value.

\begin{tabular}{|l|c|c|c|c|c|c|}
\hline Measure & Controls $^{(\mathrm{a})}$ & Parents $^{(\mathrm{b})}$ & ASD $^{(\mathrm{c})}$ & ${\text { F-statistic }(\mathrm{p}) ; \eta^{2}}^{(\mathrm{c})}-(\mathrm{a})$ & (b) \\
\hline AQ & $15.1(5.8,5-28)$ & $15.6(9.0,2-45)$ & $35.8(5.9,22-46)$ & $99.4(<.001) ; .54$ & $20.5(<.001)$ & 1.4 \\
\hline Socskill & $2.0(1.8,0-8)$ & $2.9(2.8,0-10)$ & $7.8(2.2,2-10)$ & $70.2(<.001) ; .46$ & $5.7(<.001)$ & 0.8 \\
\hline Attswitch & $3.5(1.7,1-8)$ & $3.8(2.4,0-9)$ & $8.2(1.7,3-10)$ & $65.6(<.001) ; .44$ & $4.7(<.001)$ & 0.5 \\
\hline Atttodetail & $4.8(1.9,2-9)$ & $4.0(2.3,0-10)$ & $6.6(2.1,2-10)$ & $12.4(<.001) ; .13$ & $1.8(<.001)$ & -0.3 \\
\hline Comm. & $2.2(1.8,0-7)$ & $2.5(2.4,0-9)$ & $6.9(2.0,1-10)$ & $61.9(<.001) ; .43$ & $4.7(<.001)$ & 0.3 \\
\hline Imag. & $2.6(2.2,0-10)$ & $2.4(1.9,0-8)$ & $6.3(1.6,3-9)$ & $51.4(<.001) ; .38$ & $3.6(<.001)$ & 0.1 \\
\hline BDI & $6.0(5.9,0-28)$ & $10.0(8.9,0-49.7)$ & $14.6(9.5,0-36)$ & $11.4(<.001) ; .12$ & $8.6(<.001)$ & 2.9 \\
\hline STAI-T & $37.9(11.9,20-65)$ & $54.2(13.7,20-80)$ & $42.2(12.8,26-76)$ & $18.9(<.001) ; .18$ & $16.4(<.001)$ & 3.6 \\
\hline STAI-S & $36.4(11.5,20-68)$ & $38.8(12.8,20-77)$ & $46.1(13.4,20-68)$ & $7.3(<.001) ; .08$ & $9.8(0.001)$ & 1.5 \\
\hline TAS-20 & $40.0(9.2,21-65)$ & $42.9(13.6,22-75)$ & $61.4(12.2,36-87)$ & $35.9(<.001) ; .30$ & $21.3(<.001)$ & 4.8 \\
\hline DIF & $12.2(5.0,7-25)$ & $13.7(6.4,7-30)$ & $21.6(6.8,7-34)$ & $27.1(<.001) ; .24$ & $9.3(<.001)$ & 1.7 \\
\hline DDF & $10.8(4.2,5-19)$ & $11.5(5.2,5-21)$ & $18.1(4.2,5-25)$ & $29.1(<.001) ; .26$ & $7.2(<.001)$ & 1.3 \\
\hline EOT & $16.9(5.1,8-28)$ & $17.7(4.8,8-29)$ & $21.7(5.1,10-37)$ & $10.3(<.001) ; .11$ & $4.8(<.001)$ & 1.7 \\
\hline BVAQ-B & $45.0(8.9,28-64)$ & $46.4(9.5,26-75)$ & $55.1(9.9,29-75)$ & $13.0(<.001) ; .14$ & $10.0(<.001)$ & 2.8 \\
\hline Cog & $25.4(5.1,14-36)$ & $26.7(7.5,13-43)$ & $33.8(7.2,20-47)$ & $16.8(<.001) ; .17$ & $8.4(<.001)$ & 2.3 \\
\hline Aff & $19.6(5.4,10-31)$ & $19.7(4.7,9-32)$ & $21.3(5.6,9-33)$ & $1.1(.327) ; .01$ & - & \\
\hline PAS & $14.1(11.1,0-47)$ & $18.4(13.8,1-53)$ & $24.3(8.3,9-43)$ & $8.8(<.001) ; .09$ & $10.4(<.001)$ & 7.3 \\
\hline SAS & $11.2(8.5,1-29)$ & $13.6(10.2,0-38)$ & $19.9(9.2,5-38)$ & $8.7(<.001) ; .09$ & $8.6(<.001)$ & 3.5 \\
\hline
\end{tabular}


Table 2. Percent of each group ( $n$ ) on test measures where cut-off scores for assigning participants to categories of performance are available. Chi-square comparisons for the three groups and the different performance categories are also shown.

\begin{tabular}{|c|c|c|c|c|}
\hline Scale & Controls & Parents & ASD & $\chi^{2 \text {-statistic }(p)}$ \\
\hline AQ: Typical & $91.5(43)$ & $78.2(68)$ & $2.6(1)$ & $115.8(<.001)$ \\
\hline BAP & $8.5(4)$ & $13.8(12)$ & $13.2(5)$ & \\
\hline MAP & $0.0(0)$ & $4.6(4)$ & $21.1(8)$ & \\
\hline NAP & $0.0(0)$ & $3.4(3)$ & $63.2(24)$ & \\
\hline BDI: non-depressed & $83.0(39)$ & $66.7(58)$ & $36.8(14)$ & $22.3(0.001)$ \\
\hline dysphoria & $10.6(5)$ & $12.6(11)$ & $18.4(7)$ & \\
\hline dysphoria/depression & $2.1(1)$ & $6.9(6)$ & $13.2(5)$ & \\
\hline clinical depression & $4.3(2)$ & $13.8(12)$ & $31.6(12)$ & \\
\hline TAS-20: not alexithymic & $89.4(42)$ & $73.6(64)$ & $26.3(10)$ & $48.2(<.001)$ \\
\hline intermediate & $8.5(4)$ & $12.6(11)$ & $18.4(7)$ & \\
\hline alexithymic & $2.1(1)$ & $13.8(12)$ & $55.3(21)$ & \\
\hline PAS: not anhedonic & $70.2(33)$ & $62.1(54)$ & $18.4(7)$ & $26.6(<.001)$ \\
\hline anhedonic & $29.8(14)$ & $37.9(33)$ & $81.6(31)$ & \\
\hline SAS: not anhedonic & $61.7(29)$ & $50.6(44)$ & $26.3(10)$ & $10.9(0.004)$ \\
\hline anhedonic & $38.3(18)$ & $49.4(43)$ & $73.7(28)$ & \\
\hline
\end{tabular}

Table 3. Pairwise correlations between questionnaire scores for the whole sample.

\begin{tabular}{|c|c|c|c|c|c|c|c|c|c|}
\hline & $\mathrm{AQ}$ & BDI & STAI-T & TAI-S & AS-2 & VAQ- & VAQ & VAQ-I & PAS \\
\hline & & & & & & $\operatorname{Cog}$ & Aff & Total & \\
\hline BDI & .329 & & & & & & & & \\
\hline STAI-T & .530 & .779 & . & & & & & & \\
\hline STAI-S & .390 & .787 & .821 & . & & & & & \\
\hline TAS-20 & .716 & .412 & .504 & .406 & & & & & \\
\hline BVAQ-B Cog. & .633 & .263 & .400 & .295 & .826 & . & & & \\
\hline BVAQ- B Aff. & .178 & -.223 & -.217 & -.157 & .138 & .260 & & & \\
\hline BVAQ-B Total & .560 & .083 & .189 & .141 & .684 & .875 & .695 & & \\
\hline PAS & .376 & .145 & .200 & .107 & .494 & .481 & .285 & .501 & \\
\hline SAS & .546 & .222 & .341 & .219 & .585 & .601 & .236 & .566 & .657 \\
\hline
\end{tabular}

Table 4. Cluster membership counts (\%) using BVAQ-B total (I) or BVAQ-Cognitive (II) scores in latent profile analysis.

\begin{tabular}{|c|c|c|c|c|c|}
\hline & & Controls & Parents & ASD & $N$ \\
\hline \multirow[t]{4}{*}{ I } & A & $28(42)$ & $39(58)$ & $0(0)$ & 67 \\
\hline & B & $7(30)$ & $16(70)$ & $0(0)$ & 23 \\
\hline & $\mathrm{C}$ & $11(19)$ & $25(44)$ & $21(37)$ & 57 \\
\hline & D & $1(4)$ & $7(28)$ & $17(68)$ & 25 \\
\hline \multirow[t]{4}{*}{ II } & A & $28(42)$ & $39(58)$ & $0(0)$ & 67 \\
\hline & B & $7(30)$ & $16(70)$ & $0(0)$ & 23 \\
\hline & $\mathrm{C}$ & $12(21)$ & $24(43)$ & $20(36)$ & 56 \\
\hline & D & $0(0)$ & $8(31)$ & $18(69)$ & 26 \\
\hline
\end{tabular}




\begin{tabular}{|l|l|l|l|l|}
\hline$N$ & 47 & 87 & 38 & 172
\end{tabular}

Figure 1. Distribution of questionnaire scores in each group, as smoothed density estimates.

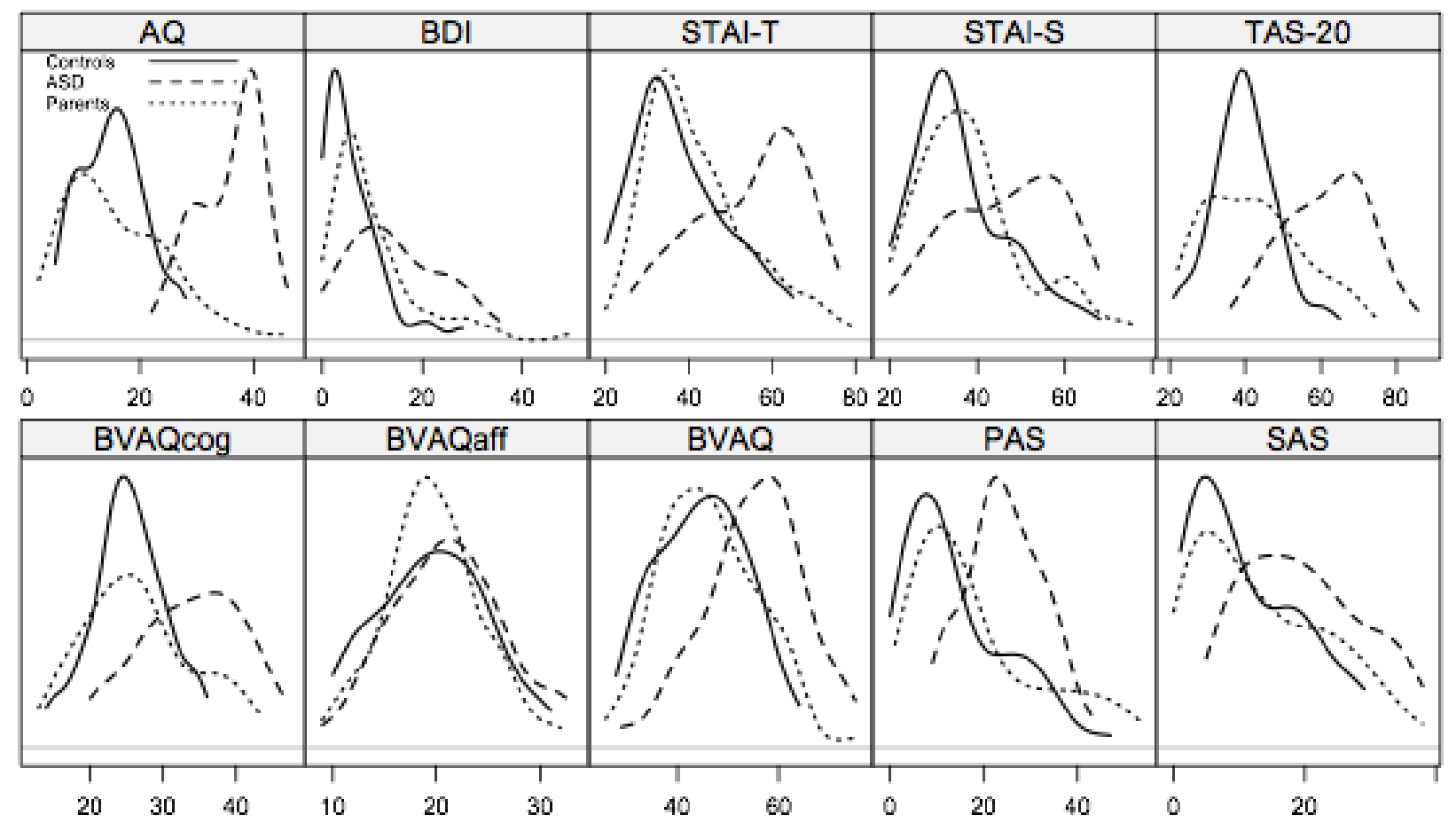

Figure 2. Top: Distribution of AQ scores by group (filled areas) and cluster membership (dotted lines), as smoothed density estimates. Bottom: Standardized trait scores by cluster. 

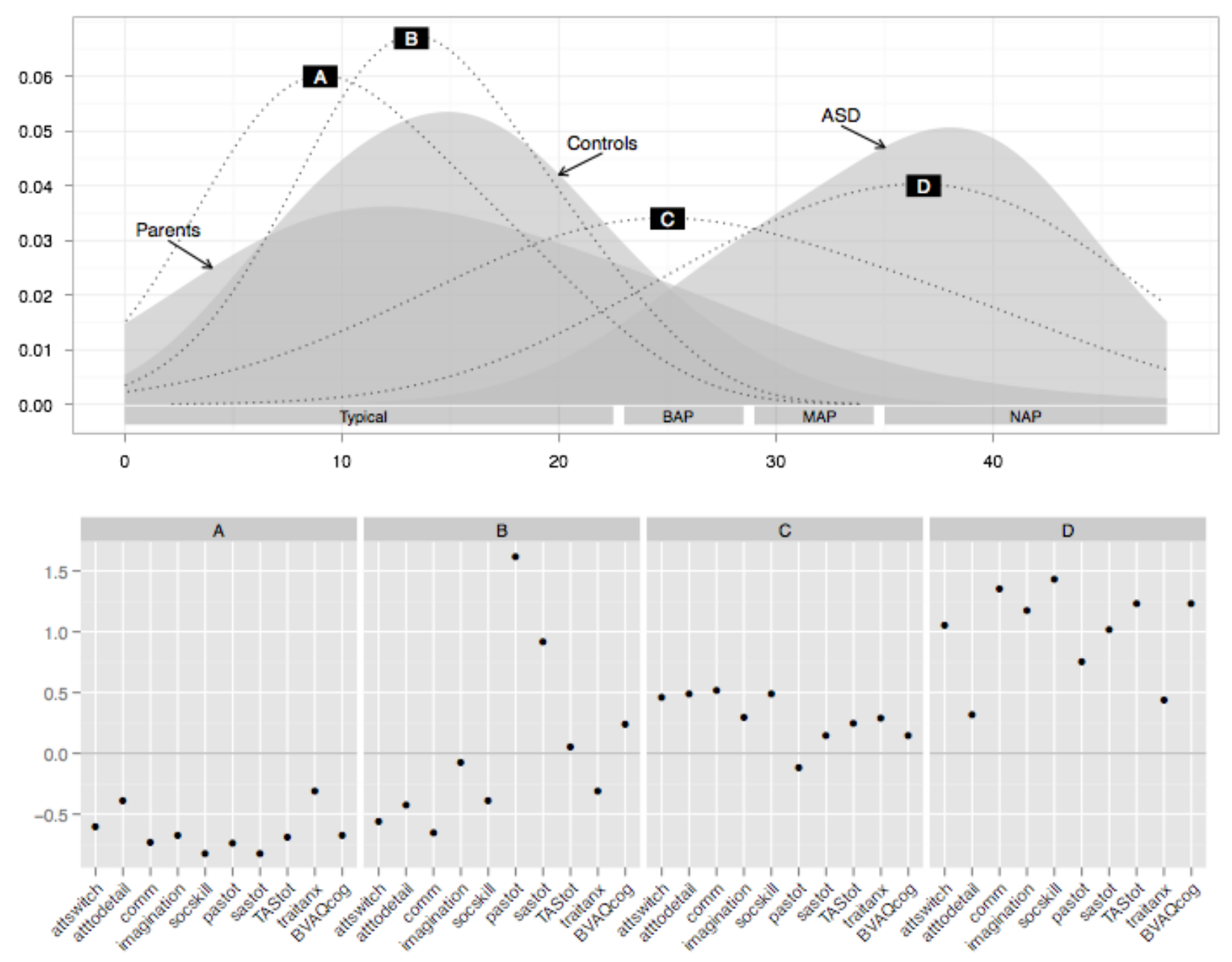

Supplementary Table 1. Between-group comparisons (a: Controls; b: Parents; c: ASD) on each test measure adjusted for age, gender, BDI and STAI-S.

\begin{tabular}{|l|c|c|c|c|}
\hline Measure & F-statistic $(\mathrm{p}) ; \eta^{2}$ & $(\mathrm{c})-(\mathrm{a})$ & $(\mathrm{b})-(\mathrm{a})$ & $(\mathrm{c})-(\mathrm{b})$ \\
\hline AQ & $83.9(<.001), 0.50$ & $18.9(<.001)$ & $1.3(0.629)$ & $17.6(<.001)$ \\
\hline Socskill & $57.2(<.001), 0.41$ & $5.3(<.001)$ & $0.8(0.186)$ & $4.5(<.001)$ \\
\hline Attswitch & $54.4(<.001), 0.40$ & $4.4(<.001)$ & $0.5(0.439$ & $3.9(<.001)$ \\
\hline Atttodetail & $8.6(<.001), 0.09$ & $1.4(0.011)$ & $-0.4(0.564)$ & $1.9(<.001)$ \\
\hline Comm. & $50.1(<.001), 0.38$ & $4.3(<.001)$ & $0.2(0.836)$ & $4.0(<.001)$ \\
\hline Imag. & $43.5(<.001), 0.35$ & $3.5(<.001)$ & $0.2(0.899)$ & $3.4(<.001)$ \\
\hline STAI-T & $8.9(<.001), 0.10$ & $6.6(<.001)$ & $1.3(0.643)$ & $5.3(0.002$ \\
\hline TAS-20 & $23.4(<.001), 0.22$ & $17.1(<.001$ & $3.7(0.240)$ & $13.4(<.001)$ \\
\hline DIF & $15.5(<.001), 0.16$ & $6.4(<.001)$ & $1.0(0.619)$ & $5.4(<.001)$ \\
\hline DDF & $18.2(<.001), 0.18$ & $5.7(<.001)$ & $0.9(0.554)$ & $4.8(<.001)$ \\
\hline EOT & $9.6(<.001), 0.10$ & $4.9(<.001)$ & $1.8(0.177)$ & $3.2(0.009)$ \\
\hline BVAQ-B & $11.7(<.001), 0.12$ & $10.1(<.001$ & $3.1(0.214)$ & $7.0(0.002)$ \\
\hline Cog & $11.1(<.001), 0.12$ & $7.1(<.001$ & $2.1(0.267)$ & $5.0(0.002$ \\
\hline Aff & $3.6(0.029), 0.04$ & $3.0(0.023)$ & $1.0(0.546)$ & $2.0(0.149)$ \\
\hline
\end{tabular}




\begin{tabular}{l|l|l|l|l|} 
PAS & $6.5(0.002), 0.07$ & $9.4(0.003)$ & $6.9(0.014)$ & $2.5(0.601)$ \\
\hline SAS & $5.3(0.006), 0.06$ & $7.1(0.004)$ & $3.2(0.225)$ & $3.9(0.134)$ \\
\hline
\end{tabular}

\begin{tabular}{|l|r|c|c|c|}
\hline Scale & F-statistic $(\mathrm{p}), \eta^{2}$ & $(\mathrm{c})-(\mathrm{a})$ & $(\mathrm{b})-(\mathrm{a})$ & $(\mathrm{c})-(\mathrm{b})$ \\
\hline AQ & $83.9(<.001), 0.50$ & $18.9(<.001)$ & $1.3(0.629)$ & $17.6(<.001)$ \\
\hline socskill & $57.2(<.001), 0.41$ & $5.3(<.001)$ & $0.8(0.186)$ & $4.5(<.001)$ \\
\hline attswitch & $54.4(<.001), 0.40$ & $4.4(<.001)$ & $0.5(0.439$ & $3.9(<.001)$ \\
\hline attodetail & $8.6(<.001), 0.09$ & $1.4(0.011)$ & $-0.4(0.564)$ & $1.9(<.001)$ \\
\hline comm & $50.1(<.001), 0.38$ & $4.3(<.001)$ & $0.2(0.836)$ & $4.0(<.001)$ \\
\hline imagination & $43.5(<.001), 0.35$ & $3.5(<.001)$ & $0.2(0.899)$ & $3.4(<.001)$ \\
\hline STAl-T & $8.9(<.001), 0.10$ & $6.6(<.001)$ & $1.3(0.643)$ & $5.3(0.002)$ \\
\hline TAS-20 & $23.4(<.001), 0.22$ & $17.1(<.001)$ & $3.7(0.240)$ & $13.4(<.001)$ \\
\hline TAS DIF & $15.5(<.001), 0.16$ & $6.4(<.001)$ & $1.0(0.619)$ & $5.4(<.001)$ \\
\hline TAS DDF & $18.2(<.001), 0.18$ & $5.7(<.001)$ & $0.9(0.554)$ & $4.8(<.001)$ \\
\hline TAS EOT & $9.6(<.001), 0.10$ & $4.9(<.001)$ & $1.8(0.177)$ & $3.2(0.009)$ \\
\hline BVAQ-B & $11.7(<.001), 0.12$ & $10.1(<.001)$ & $3.1(0.214)$ & $7.0(0.002)$ \\
\hline BVAQ cog & $11.1(<.001), 0.12$ & $7.1(<.001)$ & $2.1(0.267)$ & $5.0(0.002)$ \\
\hline BVAQ aff & $3.6(0.029), 0.04$ & $3.0(0.023)$ & $1.0(0.546)$ & $2.0(0.149)$ \\
\hline PAS & $6.5(0.002), 0.07$ & $9.4(0.003)$ & $6.9(0.014)$ & $2.5(0.601)$ \\
\hline SAS & $5.3(0.006), 0.06$ & $7.1(0.004)$ & $3.2(0.225)$ & $3.9(0.134)$ \\
\hline
\end{tabular}

Supplementary Figure a and b. Graphical summaries of the PCA results. (a) Correlation circle showing relationships between variables on the first two dimensions; screeplots of simulated random normal data were superimposed. All but one (gender) supplementary variables that were not used during the construction of the factor space are shown in grey. (b) Individual locations by group membership in the same factor space.
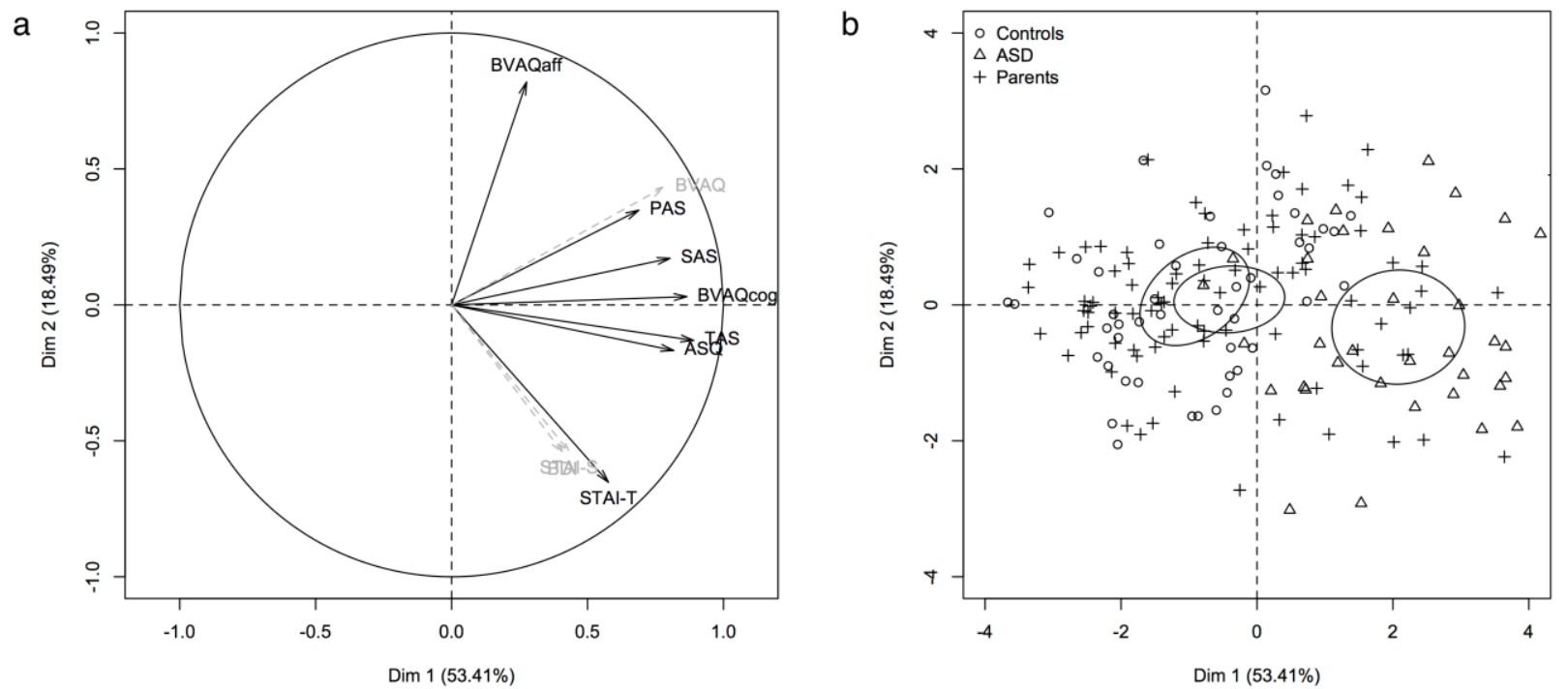


\section{REFERENCES}

Adolphs, R., Spezio, M.L., Parlier, M., Piven, J., 2008. Distinct face-processing strategies in parents of autistic children. Current Biology 18, 1090-1093.

American Psychiatric Association, 2000. Diagnostic and Statistical Manual of Mental Disorders Text Revision (DSM-IV-TR). Washington, D.C.

Assouly-Besse, F., Dollfuss, S., Petit, M., 1995. Traduction française des questionnaires d'anhédonie de Chapman: validation de la traduction française a partir de témoins et de patients schizophrènes. Encephale 22, 273-284.

Bagby, R.M., Taylor, G.J., Parker, J.D.A., 1994a. The twenty-item Toronto Alexithymia Scale-I: Item selection and cross-validation of the factor structure. Journal of Psychosomatic Research 38, 23-32.

Bagby, R.M., Taylor, G.J., Parker, J.D.A., 1994b. The twenty-item Toronto Alexithymia Scale-II: Convergent, discriminant and concurrent validity. Journal of Psychosomatic Research 38, 33-40.

Baird, G., Simonoff, E., Pickles, A., Chandler, S., Loucas, T., Meldrum, D., 2006. Prevalence of disorders of the autism spectrum in a population cohort of children in South Thames: the Special Needs and Autism Project (SNAP). Lancet 368, 210-215.

Baranek, G.T., David, F.J., Poe, M.D., Stone, W.L., Watson, L.R., 2006. Sensory Experiences Questionnaire: Discriminating sensory features in young children with autism, developmental delays, and typical development. Journal of Child Psychology and Psychiatry 47, 591-601.

Baron-Cohen, S., Ashwin, E., Ashwin, C., Tavassoli, T., Chakrabati, B., 2009. Talent in autism: hyper-systemizing, hyper-attention to detail and sensory hypersensitivity. Philosophical Transactions of the Royal Society B: Biological Sciences 364, 1377-1383.

Baron-Cohen, S., Wheelwright, S., 2003. The Friendship Questionnaire: An Investigation of adults with Asperger Syndrome or High-Functioning Autism, and normal sex differences. Journal of Autism and Developmental Disorders, 33, 509-517.

Baron-Cohen, S., Wheelwright, S., 2004. The Empathy Quotient: An Investigation of adults with Asperger Syndrome or High Functioning Autism, and normal sex differences. Journal of Autism and Developmental Disorders 34, 163-175.

Baron-Cohen, S., Wheelwright, S., Skinner, R., Martin, J., Clubley, E., 2001. The Autism-Spectrum Quotient (AQ): Evidence from Asperger Syndrome/High-Functioning Autism, males and females, scientists and mathematicians. Journal of Autism and Developmental Disorders 31, 5-17.

Beck, A., Steer, R.A., Carbin, M.G., 1988. Psychometric properties of the Beck Depression Inventory: Twenty-five years of evaluation. Clinical Psychology Review 8, 77-100.

Beck, A., Ward, C., Mendelson, M., 1961. Beck Depression Inventory (BDI). Archives of General Psychiatry 4, 561-571.

Bernhardt, B.C., Singer, T., 2012. The neural basis of empathy. Annual Review of Neuroscience $35,1-23$.

Bermond, B., 1999. Brain and alexithymia. In: Vingerhoets, A., van Bussen, F., Boelhouwers, J. (Eds.), The (non) expression of emotion in health and disease. Tilburg, the Netherlands: Tilburg University Press, pp. 115-131.

Bermond, B., Clayton, K., Liberova, A., Luminet, O., Maruszewski, T., Ricci Bitti, P.E., 2007. A cognitive and an affective dimension of alexithymia in six languages and seven populations. Cognition and Emotion 21, 1125-1136.

Berridge, K.C., Robinson, T.E., 2003. Parsing reward. Trends in Neuroscience 26(9), 507-513. Erratum in: Trends in Neuroscience 2003, 26(11), 581.

Berthoz, S., Hill, E. L., 2005. The validity of using self-reports to assess emotion regulation abilities in adults with autism spectrum disorder. European Psychiatry 20, 291-298. 
Berthoz, S., Perdereau, F., Godart, N., Corcos, M., Haviland, M.G., 2007. Observer- and self-rated alexithymia in eating disorder patients: levels and correspondence among three measures. Journal of Psychosomatic Research 62(3), 341-347.

Berthoz, S., Pouga, L., Wessa, M., 2011. Alexithymia from the Social Neuroscience Perspective. In:Decety, J., Cacioppo, J. (Eds.), Handbook of Social Neuroscience . Cambridge: Cambridge University Press, pp. 906-934.

Bird, G., Press, C., Richardson, D. C., 2011. The role of alexithymia in reduced eye-fixation in Autism Spectrum Conditions. Journal of Autism and Developmental Disorders 41, 15561564.

Bird, G., Silani, G., Brindley, R., White, S., Frith, U., Singer, T., 2010. Empathetic brain responses in insula are modulated by levels of alexithymia but not autism. Brain 133, 1515-1525.

Calder, L., Hill, V., Pellicano, E., In Press. 'Sometimes I want to play by myself': Understanding what friendship means to children with autism in mainstream primary schools. Autism 2012 Nov 27. [Epub ahead of print].

Cascio, C.J., Moana-Filho, E.J., Guest, S., Nebel, M.B., Weisner, J., Baranek, G.T., Essick, G.K., 2012. Perceptual and neural response to affective tactile texture stimulation in adults with autism spectrum disorders. Autism Research 5, 231-244.

Cederlund, M., Hagberg, B., Gillberg, C., 2010. Asperger syndrome in adolescent and young adult males. Interview, self- and parent assessment of social, emotional and cognitive problems. Research in Developmental Disabilities 31, 287-298.

Chapman, L.J., Chapman, J.P., Raulin, M.L., 1976. Scales for physical and social anhedonia. Journal of Abnormal Psychology 87, 374-407.

Chevallier, C., Grèzes, J., Molesworth, C. J., Berthoz, S., Happé, F., 2012a. Brief Report: Selective Social Anhedonia in High Functioning Autism. Journal of Autism and Developmental Disorders 42, 1504-1509.

Chevallier, C., Kohls, G., Troiani, V., Brodkin, E.S., Schultz, R.T., 2012b. The social motivation theory of autism. Trends in Cognitive Science 16, 231-239.

Crane, L., Pring, L., Jukes, K., Goddard, L,., 2012. Patterns of autobiographical memory in adults with autism spectrum disorder. Journal of Autism and Developmental Disorders 42, 21002112.

Dowd, E.C., Barch, D.M., 2012. Pavlovian reward prediction and receipt in schizophrenia: relationship to anhedonia. PloS One 7(5):e35622.

Eckblad, M.L., Chapman, L.J., Chapman, J.P., Mishlove, M., 1982. The Revised Social Anhedonia Scale. Unpublished test.

Fraley, C., Raftery, A.E., 2002. Model-based clustering, discriminant analysis, and density estimation. Journal of the American Statistical Association 97, 611-631.

Fraley, C., Raftery, A.E., 2006. MCLUST Version 3 for R: Normal mixture modeling and modelbased clustering. Technical Report No. 504. Department of Statistics, University of Washington (Revised December 2009).

Freeth, M., Bullock, T., Milne, E., 2012. The distribution of and relationship between autistic traits and social anxiety in a UK student population. Autism. Sep 17. [Epub ahead of print; doi: $10.1177 / 1362361312445511]$.

Gerdts, J., Bernier, R., 2011. The broader autism phenotype and its implications on the etiology and treatment of autism spectrum disorders. Autism Research and Treatment, Aug 17. [epub ahead of print; doi: 10.1155/2011/545901.

Geschwind, D.H., 2009. Advances in autism. Annual Review of Medicine 60,367-380.

Gottesman, I.I., Gould, T.D., 2003. The Endophenotype Concept in Psychiatry: Etymology and Strategic Intentions. American Journal of Psychiatry, 160, 636-645. 
Harvey, P.O., Armony, J., Malla, A., Lepage, M., 2010. Functional neural substrates of selfreported physical anhedonia in non-clinical individuals and in patients with schizophrenia. Journal of Psychiatric Research 44, 707-716.

Heaton P, Reichenbacher L, Sauter D, Allen R, Scott S, Hill E., 2012. Measuring the effects of alexithymia on perception of emotional vocalizations in autistic spectrum disorder and typical development. Psychological Medicine, 42, 2453-2459.

Hill, E.L., Berthoz, S., Frith, U., 2004. Brief Report: Cognitive Processing of Own Emotions in Individuals with Autistic Spectrum Disorder and in Their Relatives. Journal of Autism and Developmental Disorders 34, 229-235.

Hill, E.L., Bird, C.A., 2006. Executive processes in Asperger syndrome: Patterns of performance in a multiple case series. Neuropsychologia 44, 2822-2835.

Horan, W.P., Kring, A.M., Gur, R.E., Reise, S.P., Blanchard, J.J., 2011. Development and psychometric validation of the Clinical Assessment Interview for Negative Symptoms (CAINS). Schizophrenia Research 132, 140-145.

Hurtig, T., Kuusikko, S., Mattila, M.L., Haapsamo, H., Ebeling, H., Jussila, K., Joskitt, L., Pauls, D., Moilanen, I., 2009. Multi-informatn reports of psychiatric symptoms among highfunctioning adolescents with Asperger syndrome or autism. Autism 13, 583-598.

Kanner, L., 1943. Autistic Disturbances of Affective Contact. Nervous Child 2, 217-250.

Kätsyri, J., Saalasti, S., Tiippana, K., von Wendt, L., Sams, M., 2008. Impaired recognition of facial emotions from low-spatial frequencies in Asperger syndrome. Neuropsychologia 46, 18881897.

Kohls, G., Chevallier, C., Troiani, V., Schultz, R.T., 2012. Social 'wanting' dysfunction in autism: neurobiological underpinnings and treatment implications. Journal of Neurodevelopmental Disorders 4, 10.

Konstantareas, M.M., Stewart, K., 2006. Affect regulation and temperament in children with Autism Spectrum Disorder. Journal of Autism and Developmental Disorders 36, 143-154.

Lazarfield, P. F., Henry, N. W., 1968. Latent Structure Analysis. Boston: Houghton Mifflin.

Lombardo, M. V., Barnes, J. L., Wheelwright, S. J., Baron-Cohen, S., 2007. Self-referential cognition and empathy in autism. PloS ONE 2, e883.

Lopata, C., Toomey, J.A., Fox, J.D., Volker, M.A., Chow, S.Y., Thomeer, M.L., Lee, G.K., Rodgers, J.D., McDonald, C.A., Smerbeck, A.M., 2010. Anxiety and depression in children with HFASDs: symptom levels and source differences. Journal of Abnormal Child Psychology 38, 765-776.

Lord, C., Risi, S., 1998. Frameworks and methods in diagnosing Autism Spectrum Disorders. Mental Retardation and Developmental Disabilities Research Reviews 4, 90-96.

Lord, C., Rutter, M., DiLavore, P. S., Risi, S., 1999. The ADOS-G (Autism Diagnostic Observation Schedule - Generic). Los Angeles: Western Psychological Services.

Mason, O., Claridge, G., 2006. The Oxford-Liverpool Inventory of Feelings and Experiences (OLIFE): further description and extended norms. Schizophrenia Research 82, 203-211.

Mazefsky, C.A., Conner, C.M., Oswald, D.P., 2010. Association between depression and anxiety in high-functioning children with autism spectrum disorders and maternal mood symptoms. Autism Research 3, 120-127.

Minshew, N.J., Hobson, J.A., 2008. Sensory sensitivities and performance on sensory perceptual tasks in high-functioning individuals with autism. Journal of Autism and Developmental Disorders, 38, 1485-1498.

Petrides, K., Hudry, K., Michalaria, G., Swami, V., Sevdalis, N., 2011. A comparison of the trait emotional intelligence profiles of individuals with and without Asperger syndrome. Autism $15,671-682$. 
Poljac, E., Poljac, E., Wagemans, J., 2012. Reduced accuracy and sensitivity in the perception of emotional facial expressions in individuals with high autism spectrum traits. Autism. Sep 17. [Epub ahead of print; doi: 10.1177/1362361312455703].

Rawlings, D. B., Locarnini, A., 2008. Dimensional schizotypy, autism, and unusual word associations in artists and scientists. Journal of Research in Personality 42, 465-471.

Rieffe, C., Oosterveld, P., Terwogt, M.M., Mootz, S., van Leeuwen, E., Stockmann, L., 2011. Emotion regulation and internalizing symptoms in children with autism spectrum disorders. Autism 15, 655-670.

Robledo, J., Donnellan, A.M., Strandt-Conroy, K., 2012. An exploration of sensory and movement differences from the perspective of individuals with autism. Frontiers in Integrative Neuroscience 6, 107.

Rogers, S.J., Ozonoff, S., 2005. Annotation: what do we know about sensory dysfunction in autism? A critical review of the empirical evidence. Journal of Child Psychology and Psychiatry 46, 1255-1268.

Rogers, K., Dziobek, I., Hassenstab, J., Wolf, O., Convit, A., 2007. Who Cares? Revisiting Empathy in Asperger Syndrome. Journal of Autism and Developmental Disorders 37, 709715.

Samson, A.C., Huber, O., Gross, J.J., 2012. Emotion regulation in Asperger's syndrome and highfunctioning autism. Emotion 12, 659-665.

Schultz, R.T., 2005. Developmental deficits in social perception in autism: the role of the amygdala and fusiform face area. International Journal of Developmental Neuroscience 23, 125-141.

Silani, G., Bird, G., Brindley, R., Singer, T., Frith, C., Frith, U., 2008. Levels of emotional awareness and autism: An fMRI study. Social Neuroscience 3, 97-112.

Spielberger, C. D., 1983. State-Trait Anxiety Inventory (Form Y) manual. Mind Garden: Redwood City, CA.

Stuhrmann, A., Dohm, K., Kugel, H., Zwanzger, P., Redlich, R., Grotegerd, D., Rauch, A.V., Arolt, V., Heindel, W., Suslow, T., Zwitserlood, P., Dannlowski, U., 2012. Mood-congruent amygdala responses to subliminally presented facial expressions in major depression: associations with anhedonia. Journal of Psychiatry and Neuroscience 37. Doi : 10.1503/jpn.120060. [epub ahead of print].

Sucksmith, E., Roth, I., Hoekstra, R.A., 2011. Autistic traits below the clinical threshold: reexamining the broader autism phenotype in the 21st century. Neuropsychology Review 21, 360-389.

Szatmari, P., Georgiades, S., Duku, E., Zwaigenbaum, L., Goldberg, J., Bennett, T., 2008. Alexithymia in Parents of Children with Autism Spectrum Disorder. Journal of Autism and Developmental Disorders 38, 1859-1865.

Voos, A.C., Pelphrey, K.A., Kaiser, M.D., In Press. Autistic traits are associated with diminished neural response to affective touch. Social Cognitive and Affective Neuroscience 2012 Mar 5. [Epub ahead of print].

Vorst, H.C.M., Bermond, B., 2001. Validity and reliability of the Bermond-Vorst Alexithymia Questionnaire. Personality and Individual Differences 30, 413-434.

Wheelwright, S., Auyeung, B., Allison, C., Baron-Cohen, S., 2010. Defining the broader, medium and narrow autism phenotype among parents using the Autism Spectrum Quotient (AQ). Molecular Autism 1(10). 